Herz 2012 $\cdot 37: 719-720$

DOI 10.1007/s00059-012-3708-8

Online publiziert: 21 . Oktober 2012

(c) Urban \& Vogel 2012

\author{
A. Kribben ${ }^{1} \cdot$ R. Erbel $^{2}$ \\ ${ }^{1}$ Klinik für Nephrologie, Universitätsklinikum Essen, Universität Duisburg-Essen, Essen \\ ${ }^{2}$ Klink für Kardiologie, Westdeutsches Herzzentrum, Universitätsklinikum \\ Essen, Universität Duisburg-Essen, Essen
}

\title{
Arterielle Hypertonie
}

Über Jahrzehnte ging es bei der arteriellen Hypertonie vor allem um Grenzwerte, Blutdruckmessmethoden und die Entwicklung immer neuerer antihypertensiver Medikamente. Trotz erheblicher Erfolge ist die arterielle Hypertonie weiterhin ein wesentlicher Risikofaktor für kardiovaskuläre, zerebrovaskuläre und renale Erkrankungen und insgesamt der führende Risikofaktor der Gesamtmortalität in Deutschland. Deshalb stehen heute insbesondere die flächendeckende Erkennung und die erfolgreiche nachhaltige langfristige Behandlung der arteriellen Hypertonie im Vordergrund der präventiven Medizin.

Die arterielle Hypertonie betrifft die Hälfte aller Menschen in Deutschland. In der aktuellen populationsbasierte prospektiven Beobachtungsstudie, der Heinz Nixdorf Recall Studie, wird unter anderem die Prävalenz der arteriellen Hypertonie im Ruhrgebiet untersucht. Bei den 4443 Probanden mit vollständigen Angaben zur antihypertensiven Therapie lag der Anteil der Hypertoniker sogar bei 63\% der Männer und 52\% der Frauen. Horacek et al. beschreiben durch diese Studie erhobene weitere Details zur Prävalenz und zur geringen Therapie- und Kontrollrate der arteriellen Hypertonie.

\section{\) Die arterielle Hypertonie betrifft die Hälfte aller Menschen in Deutschland}

Für die Therapie der arteriellen Hypertonie weist J. Hoyer aus Marburg auf die Bedeutung der 4 wichtigsten nichtmedikamentösen Maßnahmen (körperliche bzw. sportliche Aktivität, Gewichtsreduktion, Diätanpassung mit Kochsalzreduktion so- wie Nikotinkarenz und mäßiger Alkoholkonsum) hin. Zur medikamentösen Therapie werden für die Medikamentengruppen Thiaziddiuretika, ACE-Hemmer, ATBlocker, Kalziumkanalblocker und Betablocker die aktuellen Erkenntnisse über Wirkungen und Nebenwirkungen beschrieben. Dabei kann die initiale Blutdrucktherapie mit einer Monotherapie begonnen werden, häufig ist jedoch eine Therapie mit mehreren Antihypertensiva erforderlich. Die Therapie muss der jeweiligen Komorbidität, wie koronare Herzkrankheit, Herzinsuffizienz, Schlaganfall, Diabetes mellitus und Niereninsuffizienz, individuell angepasst werden und kontinuierlich über viele Jahre angelegt sein.

Neben dem peripheren Blutdruck hat der zentrale Blutdruck eine besondere Bedeutung. J. Nürnberger, Schwerin, Vorstand der DeGAG (Gesellschaft für Arterielle Gefäßsteifigkeit), beschreibt, warum der zentrale Blutdruck im Alter zunimmt und warum der zentrale Blutdruck einen deutlich höheren prädiktiven Wert im Hinblick auf das Auftreten kardiovaskulärer Ereignisse hat als der periphere Blutdruck. Für die Praxis stellt Nürnberger die besten Methoden zur Messung des zentralen Blutdrucks dar und beschreibt die unterschiedlichen Wirkungen von Diuretika sowie Betablockern einerseits und ACE-Hemmern, AT-Blockern sowie Kalziumkanalblockern andererseits auf den zentralen Blutdruck und auf die Senkung des kardiovaskulären Risikos.

Einen neuen Ansatz zur Verbesserung der Therapieadhärenz und der Blutdruckkontrolle sowie lebensstiländernde Maßnahmen bei Patienten mit arterieller Hypertonie beschreiben S. Becker, Leiter der iNephro Initiative am Universitätsklinikum Essen, und Kollegen. Dabei werden Mobilapplikationen mit kombinier- ten Blutdruckmessgeräten verwendet, um die Therapie zu verbessern. Die Patienten können damit die empfohlenen Maßnahmen und deren Veränderung leicht einsehen und nachvollziehen, auf nicht eingehaltene Vorgaben wie das Vergessen einer Tabletteneinnahme hingewiesen werden sowie einfach den Blutdruck messen, speichern und ggf. weiterleiten. Die Autoren zeigen, wie dies bereits heute mit einer App erfolgen kann.

Auch bei optimaler Ausschöpfung der nichtmedikamentösen und der medikamentösen antihypertensiven Therapie kann der Blutdruck häufig nicht ausreichend gesenkt werden. Für die Patienten mit einer therapierefraktären Hypertonie wurde eine neuartige Methode, die renale Denervierung, entwickelt. Kara et al., Essen, beschreiben Erfahrungen, Indikationen, Risiken und Wirkungen des Verfahens, bei dem katheterbasiert sympathische Nervenfasern entlang der Nierenarterien abladiert werden. Neben der Blutdruckreduktion wurden zusätzliche potenzielle positive Effekte auf den Glukosestoffwechsel, das Schlafapnoesyndrom sowie die Herz- und Niereninsuffizienz beobachtet.

\section{D) Auch bei optimaler antihypertensiver Therapie kann der Blutdruck häufig nicht ausreichend gesenkt werden}

Um die Maßnahmen zu bündeln, wendet sich die Deutsche Hochdruckliga an Betroffene und Ärzte und beteiligt sich intensiv am gesundheitspolitischen Dialog. Frau A. Mitchell, Vorstand der Hochdruckliga, beschreibt die wesentlichen Faktoren einschließlich der Fortbildung 
für Ärzte und Fachpersonal mit der Möglichkeit einer Zusatzqualifikation zum Hypertensiologen DHL bzw. zum Hypertonieassistenten/-assistentin DHL, Forschungs- und Nachwuchsförderung, Motivation und Information von Selbsthilfegruppen, die Erstellung und regelmäßige Aktualisierung von Leitlinien zur Diagnostik und Therapie des Bluthochdrucks, die Vergabe von Prüfsiegeln für Blutdruckmessgeräte sowie die Zertifizierung von Hypertonie- und Präventionszentren. Auf dem 36. Jahreskongress der Deutschen Hochdruckliga wird die arterielle Hypertonie unter dem Schwerpunktthema „Innovative Konzepte zur kardiovaskulären Prävention“ in einen umfassenden Ansatz zur Prävention renaler, kardiovaskulärer und zerebrovaskulärer Erkrankungen eingebettet.

Ihre

Andreas Kribben

Raimund Erbel

\section{Korrespondenzadresse}

\section{Prof. Dr. A. Kribben}

Klinik für Nephrologie, Universitätsklinikum Essen, Universität Duisburg-Essen Hufelandstr. 55, 45122 Essen andreas.kribben@uni-due.de

\section{Hoffnung für Patienten nach Herzinfarkt}

Eine international besetzte Arbeitsgruppe von Wissenschaftlern, die von dem Direktor der Rostocker Klinik für Herzchirurgie geleitet wird, forscht seit sieben Jahren daran, wie das Herz nach einem Infarkt durch magnetgesteuerte Gen-Nanopartikel generiert werden kann.

In Rostock wurden spezielle magnetische Nanopartikel entwickelt, die mit einem Wachstumsgen zur Gefäßneubildung beladen wurden. Die magnetgesteuerten Gen-Nanopartikel, die den Patienten eingespritzt werden sollen, müssen möglichst im Herzen ankommen. Die Lösung dafür ist ein spezieller Magnet, der die Gene präzise zur Lokalisation des Herzinfarkts transportiert. Diese spezielle Methode bewirkte im Tierversuch eine deutliche Verbesserung der Herzfunktion. Mit Hilfe eines Magneten, der Ratten implantiert wurde, gelang es den Forschern durch die Gen-Nanopartikel das Wachstum von Gefäßen in Gang zu setzen und die Durchblutungsstörung des Herzens zu beheben.

Derzeit werden Nebenwirkungen untersucht, um die Sicherheit für die punktgenaue Zieltherapie am Menschen zu gewährleisten.

Quelle: Universitätsmedizin Rostock, http://www.med.uni-rostock.de 